\title{
Eradicating tumor in a recurrent cervical cancer patient with autologous tumor- infiltrating lymphocytes and a modified lymphodepleting regimen
}

Jing Guo, ${ }^{1}$ Ning Luo, ${ }^{1}$ Guihai Ai, ${ }^{1}$ Weihong Yang, ${ }^{1}$ Jihui Zhu, ${ }^{1}$ Caixia Li, ${ }^{1}$
Rong Chen, ${ }^{1}$ Changbao Zhang, ${ }^{2}$ Shupeng Liu,,${ }^{1,3}$ Huajun Jin, ${ }^{4}$ Zhongping Cheng ${ }^{1,3}$

To cite: Guo J, Luo N, Ai G, et al. Eradicating tumor in a recurrent cervical cancer patient with autologous tumor-infiltrating lymphocytes and a modified lymphodepleting regimen. Journal for ImmunoTherapy of Cancer 2022;10:e003887. doi:10.1136/jitc-2021-003887

- Additional supplemental material is published online only. To view, please visit the journal online (http://dx.doi.org/10. 1136/jitc-2021-003887).

JG and NL contributed equally. Accepted 21 December 2021

Check for updates

(C) Author(s) (or their employer(s)) 2022. Re-use permitted under CC BY-NC. No commercial re-use. See rights and permissions. Published by BMJ.

For numbered affiliations see end of article.

\section{Correspondence to Dr Zhongping Cheng; mdcheng18@263.net}

Dr Huajun Jin; hj-jin@hotmail.com

Dr Shupeng Liu; Ishup@tongji.edu.cn

\section{ABSTRACT}

Tumor-infiltrating lymphocyte (TIL) therapy has shown promising results against several cancers. However, traditional lymphodepleting regimens are severe and represent a major limitation for a more widespread use of TIL. The modified pretreatment strategies may alleviate side effects and demonstrate the persistence of tumorreactive T cells in the blood. Here, we report a case who was diagnosed recurrent cervical cancer with bladder metastasis. Omitting high dose of IL-2, she received intravenous dose of cyclophosphamide $(20 \mathrm{mg} / \mathrm{kg})$ for 3 days, approximately 48 hours before receiving the intravenous infusion of TILs. Half dosage $(100 \mathrm{mg})$ of PD1 antibody was administered with purpose of neutralizing PD1 expressed on T cells surface. She achieved complete response 10 weeks after one-time TILs infusion. Adverse reactions were negligible and safely manageable in a general ward without the need for intervention from intensive care units. Time-course peripheral blood counts and TCR repertoire sequencing demonstrated a robust expansion and long-term persistence of the infused TILs. These results illustrated the potential value of modified lymphodepletion, followed by TILs for the treatment of patients with cervical cancer with local recurrence. Trial registration number, NCT04766320.

\section{BACKGROUND}

Cervical cancer is the fourth most common malignancy in women worldwide. ${ }^{1}$ Although cervical cancer may be preventable by human papilloma virus (HPV) vaccine and early detection, ${ }^{2}$ the incidence and deaths will increase, if no action is taken. ${ }^{3}$ Chemotherapy as well as immunotherapy such as antiprogrammed death 1 receptor (antiPD1) therapy has shown clinical efficacy. ${ }^{4}$ The KEYNOTE-158 trial has contributed to the approval of pembrolizumab in recurrent programmed cell death ligand 1 (PD-L1) positive cervical cancer. ${ }^{4}$ Seventy-two trials are recruiting with anti-PD1 antibody in cervical cancer. ${ }^{5}$ However, the progress remains poor and better treatments are needed. ${ }^{6}$
Tumor-infiltrating lymphocyte (TIL) therapy, first reported in the $1980 \mathrm{~s}$, is an emerging cellular therapy with limited application to cervical cancer. TILs are mainly composed of CD3 positive $\mathrm{T}$ lymphocytes with a minor subset of natural killer cells, and target tumor cells by their relatively enriched tumor epitopespecific T cell receptors (TCRs). ${ }^{67}$ The studies, HPV-TIL cell products and LN-145 (Iovance Biotherapeutics), are evaluating two autologous cell therapies, respectively. ${ }^{8}$ Among 27 patients with metastatic cervical carcinoma who had all relapsed on the standard of treatmentschemotherapy, VEGF-targeted agents, and radiation, the objective response rate with $\mathrm{LN}-145$ was $44.4 \% .^{8}$ Traditional TIL therapy relies on high-intensity lymphodepleting pretreatment and repeated supplementary injection of highdose IL-2 to promote TIL expansion in vivo by removing inhibitory cells. ${ }^{10} 11$ Such combination of preinfusion and postinfusion treatment, however, widely causes adverse events ranging from grade 1 to grade 5 , and sometimes proves to be life-threatening for subjects demonstrating low performance status before the infusion. ${ }^{11}$ Hence an adjusted pretreatment regimen for TIL infusion is rational to alleviate its side effects.

Here, we report the clinical findings in a single patient having recurrent cervical cancer with bladder metastasis in a phase I clinical trial using autologous TIL infusion pretreated with a modified lymphodepleting regimen. The characteristic of resected tissue indicated limited lymphocytes infiltrating within tumor area. Notably, postinfusion IL-2 injection was removed from the therapy as well. The patient achieved complete response 10 weeks after TIL infusion with less adverse reactions during the therapy. Time-course peripheral blood counts and TCR repertoire sequencing demonstrated a robust expansion and long-term persistence 
A

Primary surgery plus chemotherapy

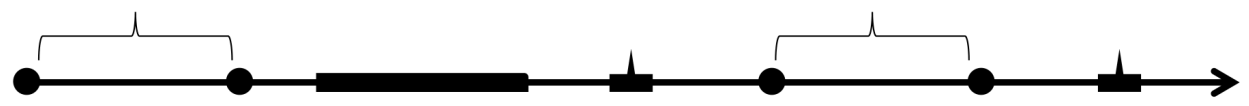

Primary diagnose
Secondary surgery plus chemotherapy
Secondary diagnose
No response enrollment

B

\begin{tabular}{cccccc}
$\begin{array}{c}\text { ICF } \\
\text { signment }\end{array}$ & $\begin{array}{c}\text { Baseline } \\
\text { assessment }\end{array}$ & Lymphodepletion & $\begin{array}{c}\text { PD1 Ab } \\
\text { TIL infusion }\end{array}$ & PD1 Ab & Assessment \\
\hline Day-11 & Day-10 & Day-5 -3 & Day0 & Day24 & Day42
\end{tabular}

C

pre-infusion

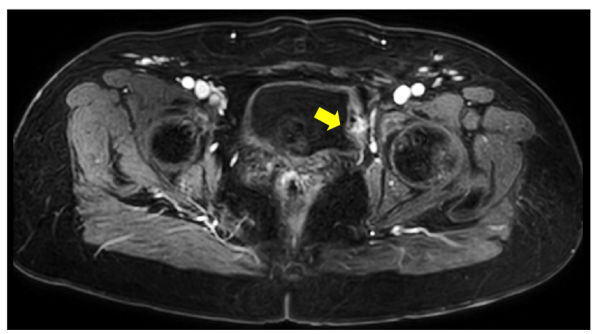

6 weeks post-infusion

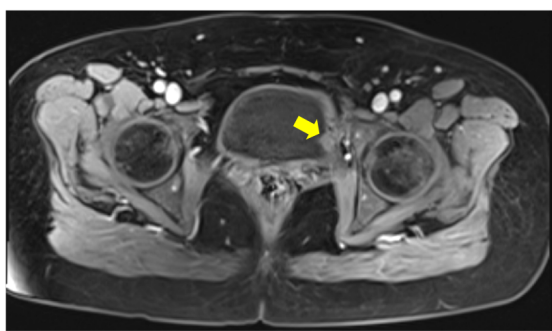

10 weeks post-infusion

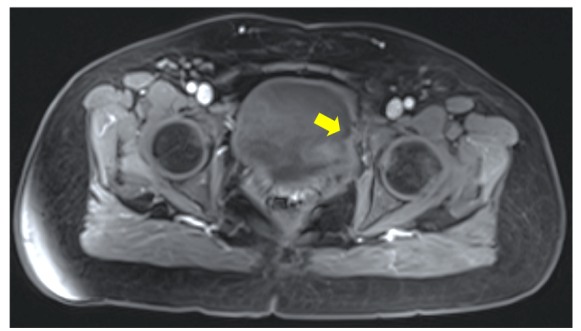

Figure 1 Clinical response. (A) Pretreatment course of this patient reported. (B) Scheme of study interventions. (C) Change of the metastatic lesions (yellow areas) before and after TIL infusion in the left bladder. TIL, tumor-infiltrating lymphocyte. PFS, progression-free survival; ICF, informed consent form.

of the infused TILs in vivo. Our results provide a novel clinical therapeutic option for solid tumors with the use of TIL infusion.

\section{CASE PRESENTATION}

\section{The medical history}

A 52-year-old woman diagnosed with recurrent cervical cancer was referred to our hospital in January, 2021 because of irregular bleeding. She was diagnosed with cervical cancer of stage IB3r based on imaging and underwent radical hysterectomy with pelvic lymphadenectomy in May, 2019. The disease stage was restratified as stage IIIC1p, which was confirmed by pelvic lymphnode involvement via pathological examination. She received four cycles of paclitaxel liposome $\left(175 \mathrm{mg} / \mathrm{m}^{2}\right.$ on day 1 of each 21-day cycle) and oxaliplatin $\left(130 \mathrm{mg} / \mathrm{m}^{2}\right.$ on day 1 of each 21-day cycle) from June 13, 2019 to August 15, 2019 and was free from disease. Seventeen months later, the disease recurred in her bladder based on magnetic resonance imaging (MRI). She underwent partial cystectomy followed by one cycle of adjuvant chemotherapy and requested tumor resection to be preserved for cellular therapy (figure 1A). All clinical parameters for this case were presented in table 1 . She requested to enroll in the present trial when disease progressed and signed the informed consent form. A timeline of interventions was depicted in figure 1B. Briefly, the patient received baseline assessment when TILs were prepared well. Then she performed lymphodepleting regimens followed by TIL infusion and PD1 antibody administration, and the first assessment happened 42 days after infusion. She did not show any symptoms of fever, or weight loss. Neither did she smoke nor use alcohol. On physical examination, she had no palpable bilateral inguinal nodes or palpable supraclavicular or axillary nodes.

Table 1 Characteristics of the patient

\begin{tabular}{|c|c|}
\hline Age & 55 \\
\hline Stage & Stage IIICp \\
\hline Therapy prior to TIL & $\begin{array}{l}\text { Radical hysterectomy+pelvic } \\
\text { lymphadenectomy, paclitaxel } \\
\text { liposome, oxaliplatin, partial } \\
\text { cystectomy, paclitaxel, carboplatin }\end{array}$ \\
\hline ECOG & 0 \\
\hline Tumor burden & $\begin{array}{l}\text { Bladder metastasis with } 3 \mathrm{~cm} \text { in } \\
\text { diameter }\end{array}$ \\
\hline State & Complete remission \\
\hline
\end{tabular}

ECOG, Eastern Cooperative Oncology Group; TIL, tumor-infiltrating lymphocyte. 


\section{Multiplexed immunofluorescence}

Pathological examination of the resected tumor tissue revealed squamous-cell carcinoma, which was consistent with her primary cervical tumor. To evaluate the perspective of tumor immune contexture, we did multiplexed immunofluorescence (mIF) analysis of the resected tumor tissue. Briefly, mIF was performed by staining $4 \mu \mathrm{m}$ thick formalin-fixed, paraffin-embedded whole tissue sections with standard using a TSA 7-color iplexed immunofluokit (D110071-50T, WiSee Bio), as previously described in other studies. ${ }^{12}$ For example, deparaffinized slides were incubated with pan-CK (AE1/AE3) primary antibody (GM351507, Gene Tech) for $30 \mathrm{~min}$, followed by the application of polymeric Horseradish peroxidaseconjugated secondary antibodies (A10011-60, Yuanxibio). IF labeling was developed for a strictly observed 10 min with Alexa Flour 488. The slides were rinsed with washing buffer after each step. These steps were repeated for the following antibodies/fluorescent dyes, in order: anti-CD68 (GM087607, Gene Tech)/TSA520, anti-CD11b (ab133357, Abcam)/TSA520, anti-CD56 (3576, Cell Signaling Technology)/TSA570, anti-CD8 (BX50036, Biolynx)/TSA670, and anti-CD3 (BX50022-C3, Biolynx) / TSA440 for panel 1; and anti-Ki-67 (BX50040, Biolynx)/ TSA520, anti-fibronectin (26836, Cell Signaling Technology)/TSA620, anti-FAP (ab207178, Abcam)/TSA570, anti-PD-L1 (13684, Cell Signaling Technology)/TSA670, and anti-CD3 (BX50022-C3, Biolynx)/TSA440 for panel 2. Each slide was then treated with 2 drops of DAPI, washed in distilled water, and manually coverslipped. Slides were air dried, mounted with anti-fade mounting medium, and taken pictures with Aperio Versa 8 tissue imaging system (Leica). The fluorescence-stained slides were scanned using a digital microscopy scanner Pannoramic MIDI tissue imaging system (3DHISTECH, Hungary). Images was analyzed using Indica Halo software. The findings showed fewer CD3 and CD8 positive cells intermingled with pan-CK positive tumor cells (figure 2A, online supplemental figure S1A, online supplemental table S1), while most CD3 positive signals colocalized with the fibroblast activation protein (FAP) positive cells. And the rate of PD-L1 in this tissue was $0.3 \%$ (figure $2 \mathrm{~B}$, online supplemental figure S1B, online supplemental table S2).

\section{Autologous TIL therapy}

TILs used in this study were provided by Shanghai Gencells Therapeutics, and manufactured according to standard Good Manufacturing Practice of Medical Products (GMP) procedure. Briefly, the tumor specimen was transferred to a GMP manufacturing facility where it was fragmented mechanically. The tumor fragments were subjected to culture in a pre-Rapid Expansion Protocol (pre-REP) medium comprizing X-VIVO 15 plus rhIL-2 (201-GMP-01M, R\&D) (2000 IU/mL), rhIL-7 (207-GMP01M, R\&D) (10 ng/mL) and rhIL-15 (247-GMP-01M, $\mathrm{R} \& \mathrm{D})(10 \mathrm{ng} / \mathrm{mL})$. TILs obtained from the pre-REP culture were incubated for 2 days in a plate precoated with anti-CD3 antibody (317302, Biolegend) and anti-CD28 (MAB342-500, R\&D) to become reactivated before they were transferred to G-REX100 harboring a REP culture medium containing X-VIVO 15 plus rhIL-2 $(300 \mathrm{IU} / \mathrm{mL})$ for a further linear expansion. The final TIL product for autologous adoptive transfer was prepared as cryopreserved drug product $(\mathrm{CDP})$ containing $1 \times 10^{10}$ cells aliquoted in $100 \mathrm{~mL}$. Before it was released, we characterized cell subtypes of the TIL product by staining cell surface markers on TILs through flow cytometry aiming to detect invigorated $\mathrm{T}$ cells (table 2, online supplemental figure S2). Additional details are provided in online supplemental files $1 ; 2$.

\section{TCR sequencing+}

Total RNA was isolated from the peripheral blood of the recipients using the RNeasy Plus Mini Kit (Qiagen). RNA

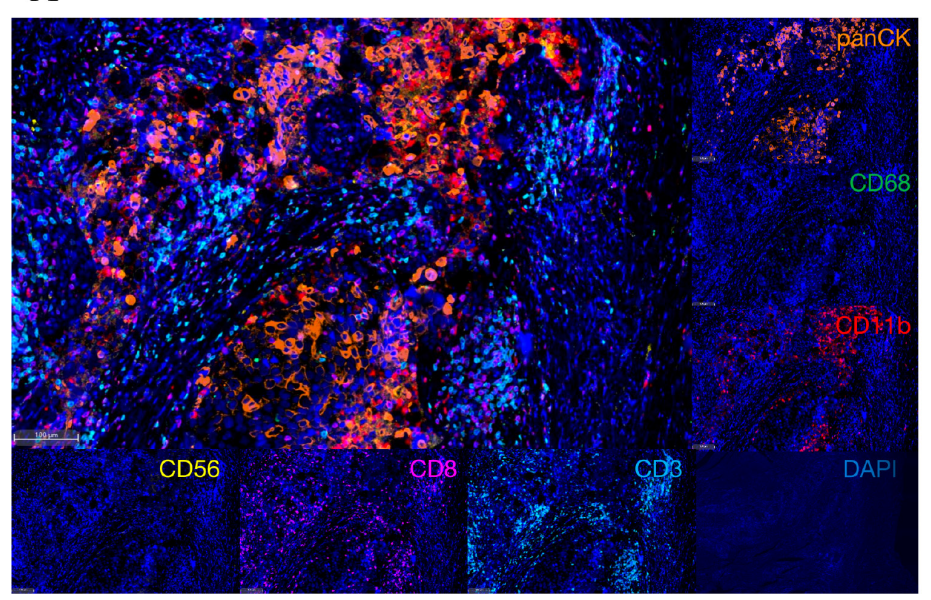

\section{B}

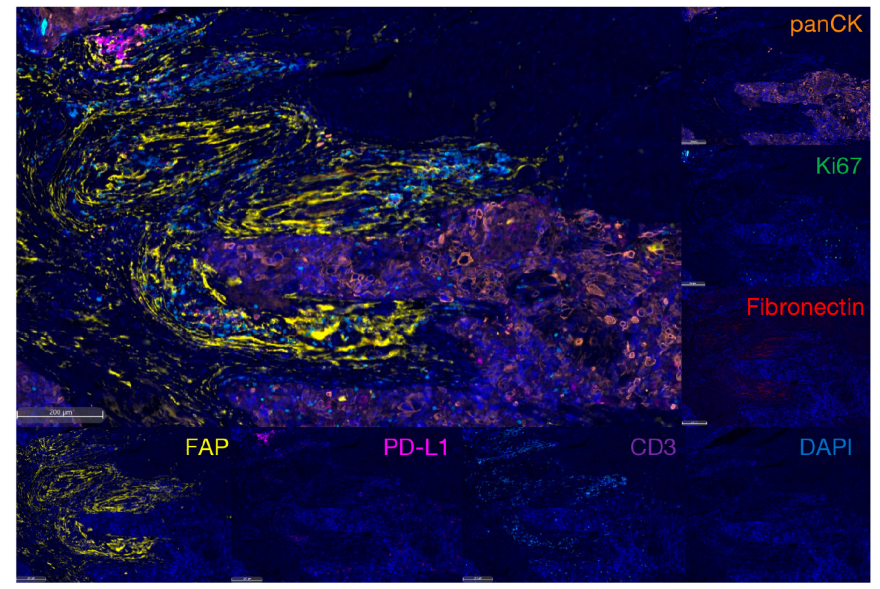

Figure 2 Multiplex immunofluorescent images of tumor tissue sections. (A) The technique labels seven channels within tumor regions as follows: pan-CK (orange), CD68 (green), CD11b (red), CD56 (yellow), CD8 (purple), CD3 (light blue), DAPI (blue). (B) Seven color demonstrates the spatial distribution of different immune lineages and markers within the stroma: pan-CK (orange), Ki67 (green), FN1 (red), FAP (yellow), PD-L1 (purple), CD3 (deep purple), DAPI (blue). CD, cluster of differentiation; FN1, fibronectin; FAP, fibroblast activation protein; PD-L1, programmed death ligand 1. 


\begin{tabular}{ll}
\hline Table 2 The characterization of TILs & \\
\hline Markers & Percentage \\
\hline CD45+CD3+ (\%Cells) & 87.75 \\
CD45+ (\%Cells) & 99.99 \\
CD56+ (\%CD45) & 30.19 \\
CD19+ (\%CD45) & 0.01 \\
CD4+ (\%CD3) & 25.97 \\
CD8+ (\%CD3) & 34.14 \\
\hline
\end{tabular}

TIL, tumor-infiltrating lymphocyte.

samples were analyzed by NGS for TRBs using the TCR profiling system. Briefly, a 5' rapid amplification of cDNA ends (RACE) unbiased amplification protocol was used. ${ }^{13}$ One common forward adaptor primer and one reverse primer corresponding to the constant (C) regions of each of the TCR $\beta$ were designed to facilitate PCR amplification of cDNA sequences in a less biased manner. This protocol uses unique molecular identifiers (UMIs) introduced in the course of cDNA synthesis to eliminate PCR and sequencing errors. Sequencing was performed on an Illumina NovaSeq system with PE150 mode.

The UMI attached to each raw sequence reads were applied to correct PCR and sequencing errors correction and PCR duplicates removing. Map V, D, J and C segments with IMGT and then extract CDR3 regions and assemble clonotype for all clones. The resulting nucleotide and amino acid sequences of CDR3 of TCR $\beta$ were determined and those with out-of-frame and stop codon sequences were removed from the identified TCR $\beta$ repertoire. We further defined amounts of each TCR $\beta$ clonotype by adding numbers of TCR $\beta$ clones sharing the same nucleotide sequence of CDR3.

\section{Clinical findings}

The patient was the first one enrolled in this study in April, 2021 meeting with inclusion criteria for recurrence of gynecological cancers and measurable lesions. Evaluations using MRI based on the enhanced-T1-weighted (T1WI) sequence showed no other disease except a mass that was $3 \mathrm{~cm}$ in diameter in her left bladder (day 10). Before TIL infusion, the patient received intravenous dosing of cyclophosphamide ( $20 \mathrm{mg} / \mathrm{kg}$ of body weight) for 3 days (day $5-3$ ). Forty-eight hours after the last dosing of cyclophosphamide, the intravenous infusion of TILs of $2 \times 10^{10}$ cells was carried out in a general ward over 60 min (day 0). A PD1 antibody drug (Sintilimab, Innovent) reduced by half dosage $(100 \mathrm{mg})$ as compared with the normal dosage was administered intravenously on the same day ( $d$ ay 0 ) before TIL infusion with purpose of neutralizing cell-surface expressed PD1. A second intravenous injection of $100 \mathrm{mg}$ of sintilimab was given 24 days after the infusion (day 24), as observable PD1 expression increased in CD3 + T cell population, to contain the rising immunosuppressive condition. Six weeks after TIL infusion, T1WI showed remarkable shrinkage of the lesion (yellow arrowheads). According to the Response Evaluation Criteria in Solid Tumors v1.1 (RECIST), ${ }^{14} \mathrm{a}$ complete response was confirmed 10 weeks after infusion (figure 1C).

To evaluate the effect of cyclophosphamide and the side effects of TIL infusion, counts of immune cells and cytokines were assessed using peripheral venous blood samples collected at multiple time points during the course of TIL therapy. Significant decrease of total white blood cell count (WBC), neutrophil count and lymphocyte count were observed after cyclophosphamide administration. Fourteen days after lymphodepleting pretreatment, the total WBC and neutrophil count gradually dropped to minima of $0.99 \times 10^{9} / \mathrm{L}$ and $0.18 \times 10^{9} / \mathrm{L}$ from $5.29 \times 10^{9} / \mathrm{L}$, and $3.79 \times 10^{9} / \mathrm{L}$, respectively. Both total $\mathrm{WBC}$ and neutrophil counts remained at a relatively low level for 16 days (figure 3A). The lymphocyte count decreased to minina of $0.28 \times 10^{9} / \mathrm{L} 6$ days after lymphodepletion and increased noticeably from day 1 to day 11. The total WBC, absolute neutrophil and lymphocyte level remained stable from 15 days after infusion (figure 3B). Interestingly, the mutually complemented change in CD4+ and CD8+ cell frequencies permanently increased the proportion of the systemic CD8+ T cells of the following blood time points (figure 4A), reflecting the activation and long-term persistent effects of TIL infusion.

Levels of cytokines, including IL-2, IL-4, IL-6, IL-8, IL-10, TNF- $\alpha$ and IFN- $\gamma$ were monitored with flow cytometry using a CBA cytokine detection kit (BD Biosciences). It showed that most of these cytokines excluding IL-6 and IL-8 maintained stable base level in peripheral blood. IL-6 level was increased on Day 11 and dropped to base level after then, while IL-8 increased dramatically on day 11 , peaked on day 26 and dropped to base level almost on day 30 (figure 3C). Additionally, hemoglobin level and platelet count were also measured. Stable with minor to moderate fluctuations were observed in both hemoglobin level and platelet count (figure 3D). No other adverse effects were observed in this patient, which confirmed that this new lymphodepletion therapy is safe enough to be performed in a ward.

To estimate the expansion of infused TILs, we used 5' RACE approach to quantify the expected frequency of TCR $\beta$ clonotype sharing the same nucleotide sequence of CDR3. ${ }^{13}$ A high frequency of CDR3 sharing had been observed on 31 days after infusion $(4.45 \%)$, nearly fourfold increase compared with the frequency of CDR3 sharing on the first day of infusion (1.28\%), suggesting that the infused TILs had profoundly changed the systemic TCR clonotype diversity (figure 5A). We further quantified similarity of the frequency distribution of CDR3 length from two samples of different time points after infusion using the Morisita-Horn (MH) similarity index, which revealed a higher $\mathrm{MH}$ value for the two samples of two closer time points (figure 5B). A timecourse $\mathrm{MH}$ value curve calculated using different timepoint samples showed a gradual decrease of similarity to 

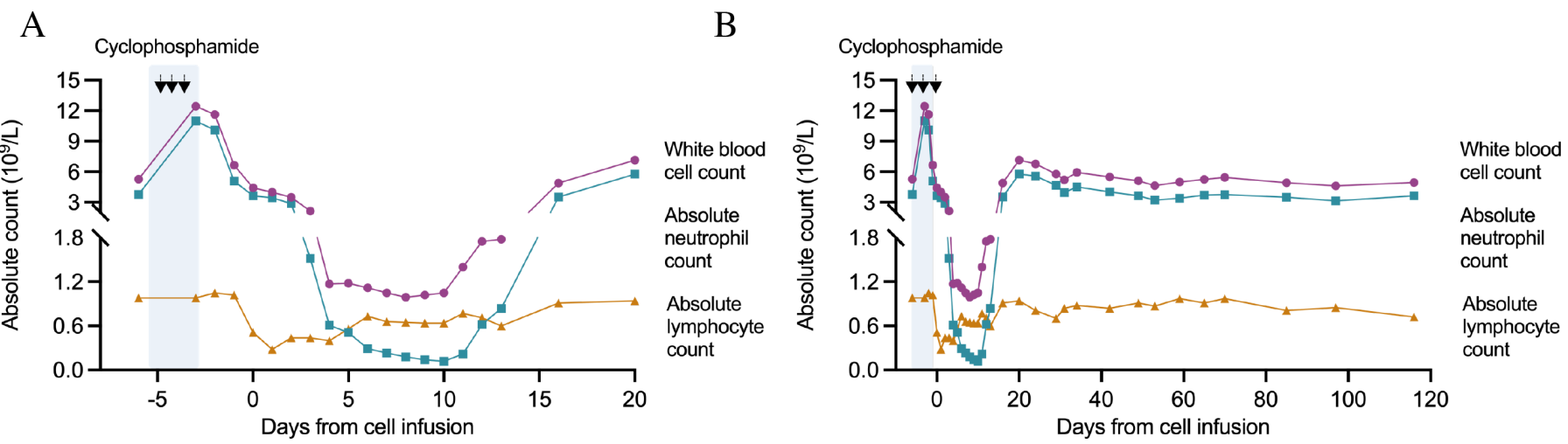

$\mathrm{C}$

$\mathrm{D}$
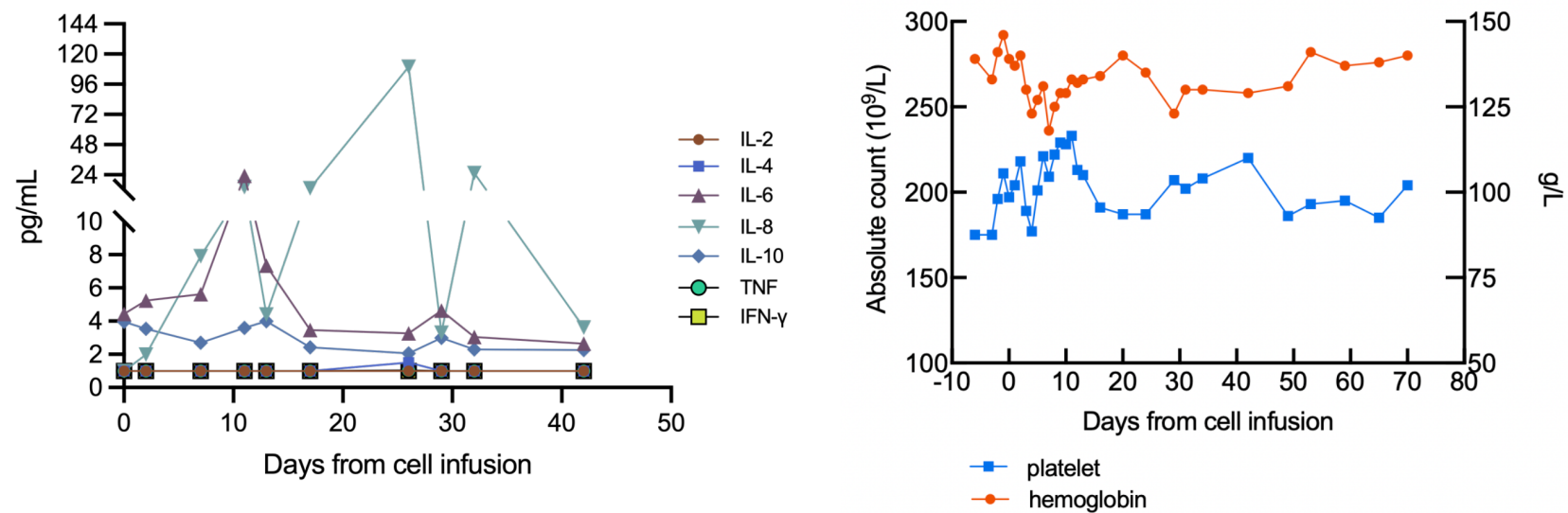

Figure 3 Change of peripheral blood cells and cytokines during treatment. (A-B) Changes of absolute count of cells in blood after lymphodepletion and TIL infusion. Dots represent white cell count; squares mean neutrophils and triangles indicate lymphocytes. (C) Cytokine dynamics after TIL infusions (from day 0 to day 42). Concentrations of IL-2, 4, 6, 8, 10, TNF and IFN- $\gamma$ were measured by flow cytometry from day 0 to day 42 . Values $\leq 1 \mathrm{pg} / \mathrm{mL}$ were marked as $1 \mathrm{pg} / \mathrm{mL}$. (D) Fluctuations of hemoglobin level and platelet count during treatment. TNF, tumor necrosis factor; IFN- $\gamma$, interferon $\gamma$.

nearing the preinfusion similarity on 59 days after infusion (figure 5C), while the time-course curve of frequency of CDR3 sharing between infusion product and TCR different time point samples after infusion showed a completely different pattern with multiple downs and falls (figure 5D). The two highest frequencies of CDR3 sharing were $27.51 \%$ and $28.11 \%$ on 2 days and 31 days after infusion, respectively. To determine the relationship between frequency of clonotype sharing and absolute count of lymphocytes, we compared the two datasets which demonstrated frequency of clonotype sharing
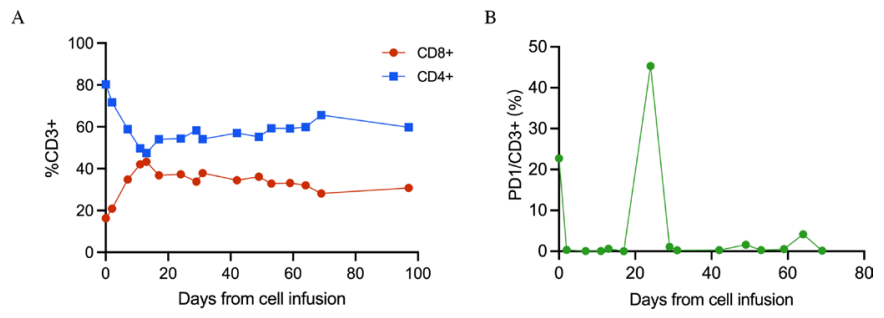

Figure 4 The change of CD4+ and CD8+ cell and PD1 expression. (A) The mutually complemented change in CD4+ and CD8+ cell frequencies increased the proportion of the systemic CD8+ T cells. (B) PD1 expression on TILs increased on 24 days after infusion. was highly coincident with absolute count of lymphocytes from peripheral blood (figure 5D). These findings suggested a robust expansion of infused TILs and a permanent change to the systemic peripheral blood $\mathrm{T}$ lymphocyte profile.

\section{DISCUSSION AND CONCLUSION}

TIL therapy using in vitro expanded naturally occurring tumor-residing lymphocytes has produced durable, complete regression in patients with cervical cancer. ${ }^{9}$ For clinic use, large numbers of antitumor lymphocytes (up to $10^{11}$ ) can be readily grown in vitro and selected for high-avidity tumor antigens and mediation of cancer regression. ${ }^{1115}$

Lymphodepletion using high dose chemotherapeutic drugs is the currently established pretreatment for most TIL therapies, which is believed to be able to improve the efficacy of TIL infusion by creating a favorable environment in vivo. The underlying mechanisms of lymphodepletion may include the clearance of homeostatic cellular cytokine sinks and the removal of Tregs to enhance the efficacy of transferred TILs. ${ }^{16}$ The most frequently used pretreatment regimen consists of cyclophosphamide 
A

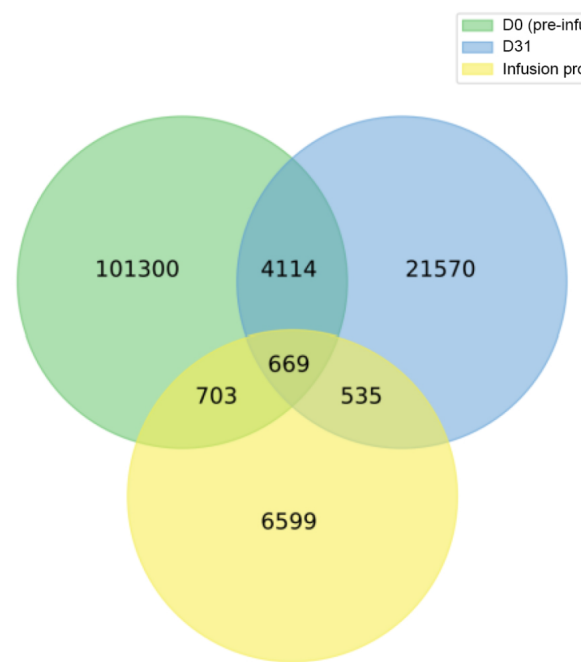

$\mathrm{C}$

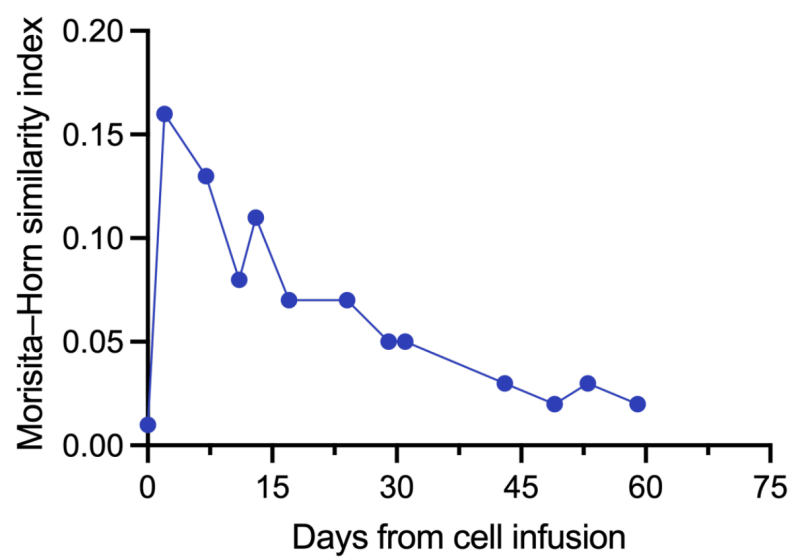

B

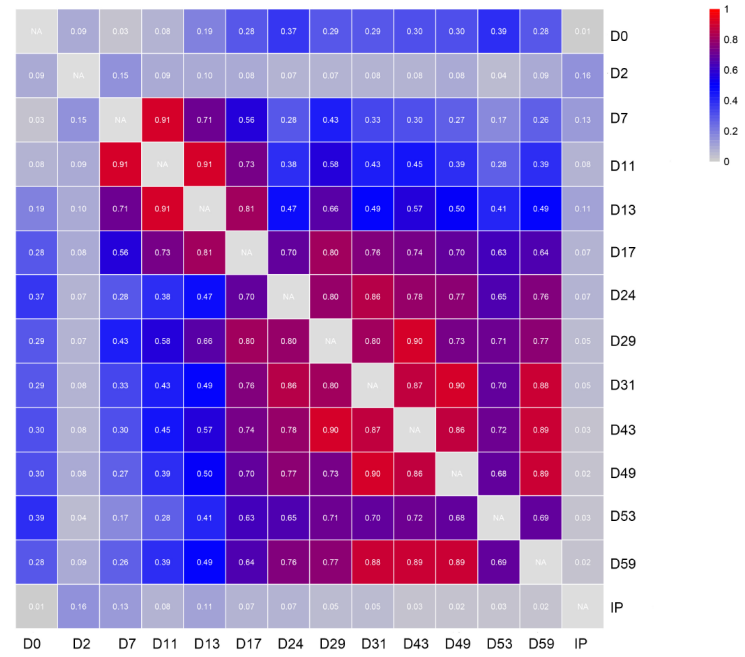

$\mathrm{D}$

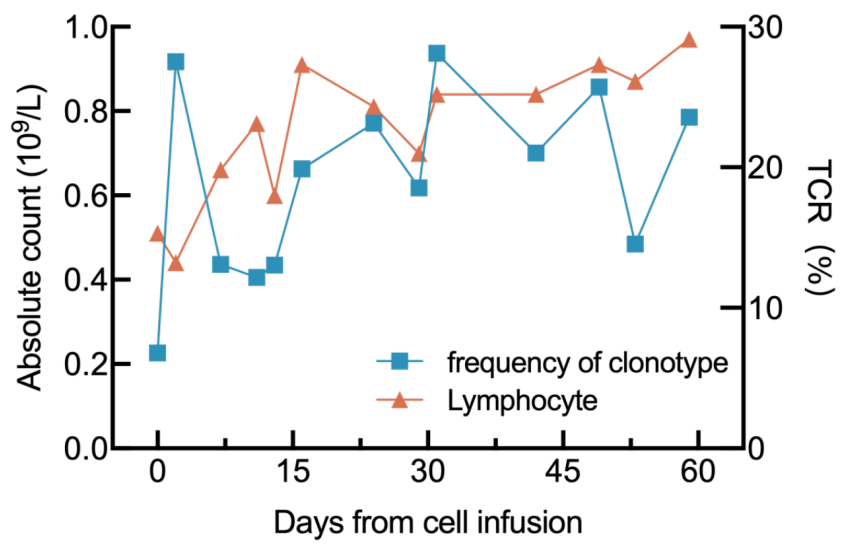

Figure 5 Shared clonotypes from samples of different time points after infusion. (A) Venn diagram of shared clonotype frequency. (B) Clustered distance matrix of subjects, using pairwise Morisita-Horn (MH) similarity of CDR3 as the distance measure. (C) A time course MH value curve using different time point samples. (D) The relationship between frequency of clonotype sharing and absolute count of lymphocytes from peripheral blood. TCR, T cell receptor.

$(60 \mathrm{mg} / \mathrm{kg})$ given for 2 days and fludarabine $(25 \mathrm{mg} /$ $\mathrm{m}^{2}$ ) administered over 5 days, followed by TIL infusion and IL-2 administration. ${ }^{17}$ Traditional pretreatment and post-TIL infusion regimen lead to numerous adverse events such as constitutional symptoms like fever and chills, as well as side effects of cardiovascular, pulmonary, renal, gastrointestinal, neurologic, dermatological, and endocrine systems. ${ }^{18-20}$ We modified the lymphodepleting regimen with half of the reported dosage of cyclophosphamide and excluded fludarabine and IL-2. As expected, on the patient treated with this modified lymphodepleting regimen in the present case showed negligible adverse reactions and achieved complete response. This finding supported the feasibility of our novel pretreatment, at least in this cervical cancer patient.

Following TILs infiltration, the killing ability of T cells might be impaired during a prolonged T cell-tumor interaction referred as $\mathrm{T}$ cell exhaustion. ${ }^{21} \mathrm{~T}$ cell exhaustion is typically characterized by reduced effector cytokines, coupled with the upregulation of multiple inhibitory receptors such as PD1, LAG-3 and TIM-3, as well as loss an effective attack on the target cells. ${ }^{1022}{ }^{23}$ Without high dose lymphodepleting pretreatment coupled with high dose IL-2 utilization after infusion, TILs are more prone to be suppressed by immunosuppressive tumor microenvironment. Blockading immune checkpoints such as PD1 and CTLA-4, has proven to rescue T lymphocytes from immunosuppressive condition. ${ }^{10}$ The exhaustion of TIL was assessed of the following blood time points by PD-1 level. Based on PD1 expression on TILs (figure 4B), an approved anti-PD1 antibody, sintilimab, was used in a half-reduced dosage as compared with the labelrecommended dose with purpose of neutralizing PD1 expression on TILs, and no adverse effects related to the PD1 mAb drug was observed in this case. Additionally, most CD3 positive signals were observed in the extracellular matrix of the tumor, while only a minor proportion residing in the pan-CK positive area (figure 2), indicating the tumor may result from a failure of immune recognition or region-specific barriers to infiltration. ${ }^{24}$ The tissue architecture is preserved to improve biomarker discovery and assessment. TCR clone frequency of the infused TILs was monitored by TCR repertoire sequencing, indicating the repertoires from different time point samples were altered by new immunological encounters. ${ }^{13}$ It showed that the TCR clone frequency increased along with time 
after infusion (figure 5D) and that the enlarged proportion of CD8+ T cells lasted as long as 100 days after therapy (online supplemental figure S2A). These findings suggested a robust expansion of infused TILs and a permanent change to the systemic peripheral blood $\mathrm{T}$ lymphocyte profile even though IL-2 was not used after infusion. It is possible that IL-2 secreted by infused TILs involved in the expansion. Moreover, a similarity comparison between TCR samples from various postinfusion time points and the infusion product showed that the similarity descended continuously from 2 days post infusion. Study has demonstrated that clones expandation after infusion mainly originated from infused clusters with higher expression of cytotoxicity and proliferation genes. ${ }^{25}$ Taken together, we hypothesized that the infused TILs underwent tumor neoantigen response screening in vivo and as a result, relatively fewer numbers of TCRs, possibly tumor responsive, became enriched. Still, there is a need for more comprehensive immune biomarker analysis in the patient to identify important phenotypic and functional TIL markers as well as the sustained monitoring of persisting TIL subsets in the blood after infusion to further delineate the differential role of CD4+vs CD8+ $\mathrm{T}$ cells.

In conclusion, our study provides a promising therapeutic approach for TIL therapy in which mild chemotherapeutic pretreatment is required and post-infusion IL-2 administration is removed completely. This case represented another option for patients receiving TIL infusion. Notably, more patients and other solid tumors are needed to validate the therapeutic efficacy achieved in this case.

\section{Author affiliations \\ 'Department of Obstetrics and Gynecology, Shanghai Tenth People's Hospital, School of Medicine, Tongji University, Shanghai, People's Republic of China ${ }^{2}$ Department of Radiology, Shanghai Tenth People's Hospital, School of Medicine, Tongji University, Shanghai, People's Republic of China \\ ${ }^{3}$ Gynecologic Minimally Invasive Surgery Research Center, School of Medicine, Tongji University, Shanghai, People's Republic of China \\ ${ }^{4}$ Gencells Therapeutics, Shanghai, People's Republic of China}

Acknowledgements The authors would like to thank the patient who participated in the trial. The authors would also like to thank the physicians, nurses, research coordinators and other staff at the hospital and Binghui Zhao from Department of Radiology who assisted with the study. The authors would like to acknowledge Shanghai Gencells Therapeutics team for their contributions.

Contributors ZC, HJ and SL designed and led the clinical trial; JG, NL, GA, WY, $\mathrm{CL}$ and $\mathrm{RC}$ provided patient care; JG analyzed data and wrote the manuscript; ZC, HJ and SL designed the TIL product and in vitro experiments; JG, NL and SL interpreted data and edited the manuscript; JZ provided clinical assistance; CZ provided MR images.

Funding This work was supported by grant from the National Natural Sciences Foundation of China (82103337; to JG) and Shanghai Biomedical Science and Technology Project (21S11906600; to HJ).

Competing interests None declared.

Patient consent for publication Consent obtained directly from patient(s)

Ethics approval This study was approved by the institutional review board of Shanghai Tenth People's Hospital (IRB No. SHSY-IEC-4.0/20-238/02).

Provenance and peer review Not commissioned; externally peer reviewed.

Supplemental material This content has been supplied by the author(s). It has not been vetted by BMJ Publishing Group Limited (BMJ) and may not have been peer-reviewed. Any opinions or recommendations discussed are solely those of the author(s) and are not endorsed by BMJ. BMJ disclaims all liability and responsibility arising from any reliance placed on the content. Where the content includes any translated material, BMJ does not warrant the accuracy and reliability of the translations (including but not limited to local regulations, clinical guidelines, terminology, drug names and drug dosages), and is not responsible for any error and/or omissions arising from translation and adaptation or otherwise.

Open access This is an open access article distributed in accordance with the Creative Commons Attribution Non Commercial (CC BY-NC 4.0) license, which permits others to distribute, remix, adapt, build upon this work non-commercially, and license their derivative works on different terms, provided the original work is properly cited, appropriate credit is given, any changes made indicated, and the use is non-commercial. See http://creativecommons.org/licenses/by-nc/4.0/.

\section{REFERENCES}

1 Cohen PA, Jhingran A, Oaknin A, et al. Cervical cancer. Lancet 2019;393:169-82.

$2 \mathrm{Hu} \mathrm{Z}, \mathrm{Ma} \mathrm{D}$. The precision prevention and therapy of HPV-related cervical cancer: new concepts and clinical implications. Cancer Med 2018;7:5217-36.

3 Arbyn M, Weiderpass E, Bruni L, et al. Estimates of incidence and mortality of cervical cancer in 2018: a worldwide analysis. Lancet Glob Health 2020;8:e191-203.

4 Chung HC, Ros W, Delord J-P, et al. Efficacy and safety of pembrolizumab in previously treated advanced cervical cancer: results from the phase II KEYNOTE-158 study. J Clin Oncol 2019;37:1470-8.

5 Clinicaltrials.gov. Available: https://clinicaltrials.gov/ct2/results?term= $\mathrm{PD} 1 \&$ cond $=$ Cervical+Cancer\&Search $=$ Apply\&recrs $=a \& a g e \_v=\&$ gndr=\&type $=\& r s \mid t=$

6 Wendel Naumann R, Leath CA. Advances in immunotherapy for cervical cancer. Curr Opin Oncol 2020;32:481-7.

7 Sarnaik AA, Hamid O, Khushalani NI, et al. Lifileucel, a tumorinfiltrating lymphocyte therapy, in metastatic melanoma. J Clin Oncol 2021;39:2656-66.

8 XAmir Jazaeri A A, Amaria RN. Safety \& efficacy of adoptive cell transfer using autologous tumor infiltrating lymphocytes (LN-145) for treatment of recurrent, metastatic, or persistent cervical carcinoma. $J$ Clin Oncol 2019;37.

9 Stevanović S, Helman SR, Wunderlich JR, et al. A phase II study of tumor-infiltrating lymphocyte therapy for human papillomavirusassociated epithelial cancers. Clin Cancer Res 2019;25:1486-93.

10 Restifo NP, Dudley ME, Rosenberg SA. Adoptive immunotherapy for cancer: harnessing the T cell response. Nat Rev Immunol 2012;12:269-81.

11 Creelan BC, Wang C, Teer JK, et al. Tumor-infiltrating lymphocyte treatment for anti-PD-1-resistant metastatic lung cancer: a phase 1 trial. Nat Med 2021;27:1410-8.

12 Sun Y-F, Wu L, Liu S-P, et al. Dissecting spatial heterogeneity and the immune-evasion mechanism of CTCs by single-cell RNA-seq in hepatocellular carcinoma. Nat Commun 2021;12:4091.

13 Briney B, Inderbitzin A, Joyce C, et al. Commonality despite exceptional diversity in the baseline human antibody repertoire. Nature 2019;566:393-7.

14 Schwartz LH, Litière S, de Vries E, et al. RECIST 1.1-Update and clarification: from the RECIST Committee. Eur $J$ Cancer 2016;62:132-7.

15 Leko V, Rosenberg SA. Identifying and targeting human tumor antigens for T cell-based immunotherapy of solid tumors. Cancer Cell 2020;38:454-72.

16 Gattinoni L, Finkelstein SE, Klebanoff CA, et al. Removal of homeostatic cytokine sinks by lymphodepletion enhances the efficacy of adoptively transferred tumor-specific CD8+ T cells. J Exp Med 2005;202:907-12.

17 Chandran SS, Somerville RPT, Yang JC, et al. Treatment of metastatic uveal melanoma with adoptive transfer of tumourinfiltrating lymphocytes: a single-centre, two-stage, single-arm, phase 2 study. Lancet Oncol 2017;18:792-802.

18 Marabondo S, Kaufman HL. High-dose interleukin-2 (IL-2) for the treatment of melanoma: safety considerations and future directions. Expert Opin Drug Saf 2017;16:1347-57.

19 Mayor P, Starbuck K, Zsiros E. Adoptive cell transfer using autologous tumor infiltrating lymphocytes in gynecologic malignancies. Gynecol Oncol 2018;150:361-9.

20 Besser MJ, Shapira-Frommer R, Treves AJ, et al. Clinical responses in a phase II study using adoptive transfer of short-term cultured 
tumor infiltration lymphocytes in metastatic melanoma patients. Clin Cancer Res 2010;16:2646-55.

21 O'Donnell JS, Teng MWL, Smyth MJ. Cancer immunoediting and resistance to T cell-based immunotherapy. Nat Rev Clin Oncol 2019;16:151-67.

22 Parkhurst MR, Robbins PF, Tran E, et al. Unique neoantigens arise from somatic mutations in patients with gastrointestinal cancers. Cancer Discov 2019;9:1022-35.
23 Chen L, Flies DB. Molecular mechanisms of T cell co-stimulation and co-inhibition. Nat Rev Immunol 2013;13:227-42.

24 Zhang AW, McPherson A, Milne K, et al. Interfaces of malignant and immunologic clonal dynamics in ovarian cancer. Cell 2018;173:e22:1755-69.

25 Sheih A, Voillet V, Hanafi L-A, et al. Clonal kinetics and single-cell transcriptional profiling of CAR-T cells in patients undergoing CD19 CAR-T immunotherapy. Nat Commun 2020;11:219. 